\title{
Lentigo Maligna on a Difficult Location of the Face Treated with 5\% Imiquimod: A Case Report of 2-Year Follow-Up
}

\author{
Magdalena Misiak-Galazka (D) · Joanna Czuwara • Adam Galazka • \\ Anna Czaplicka · Lidia Rudnicka
}

Received: October 25, 2021 / Accepted: December 10, 2021 / Published online: January 26, 2022

(C) The Author(s) 2022

\section{ABSTRACT}

Topical imiquimod is an off-label alternative treatment for lentigo maligna used when surgery cannot be performed. Severe inflammatory response induced by this drug may generate many complaints and force patients to discontinue use. We present a case in which interval treatment with $5 \%$ topical imiquimod was implemented for severe inflammatory response. An 82-year-old Caucasian woman presented with a large, irregularly pigmented lesion on her left cheek within the scar of a previously excised melanocytic lesion diagnosed as junctional nevus. Based on dermatoscopical examination confirmed by histopathological description, lentigo maligna was diagnosed. Since the lesion was large and covered the lower left eyelid and due to the risk of disfigurement, the patient refused surgical excision. Therefore, treatment with imiquimod 5\% once daily, five times per week, was offered. After 5 weeks, the treatment was stopped because of intense inflammatory

M. Misiak-Galazka (ه) · J. Czuwara · A. Czaplicka . L. Rudnicka

Department of Dermatology, Medical University of Warsaw, Koszykowa 82A, 02-008 Warsaw, Poland e-mail: magdamisiak@o2.pl

\section{A. Galazka}

Department of Head and Neck Cancers, The Maria Sklodowska-Curie National Research Institute of Oncology, Warsaw, Poland reaction and ulceration. On the follow-up visit after 3 months, videodermatoscopical examination revealed changes in the pigmentation of the lentigo maligna including the presence of residual gray dots and fading of the previous dark brown and black colors. The inflammatory response had almost resolved. The second course of treatment with imiquimod five times a week was implemented again for 5 weeks, and after a 2-month interval the third course with the same regimen was started. Total clearance of the lesion was achieved, which was confirmed by videodermatoscopical examination. During the 2-year follow-up, no relapse was observed based on dermatoscopical examination. We propose interval treatment with topical imiquimod 5\% lentigo maligna for severe inflammatory reactions in patients with contraindications to surgery. This could help patients overcome this typical response effect and decrease their cessation of treatment.

Keywords: Case report; Imiquimod; Lentigo maligna 


\section{Key Summary Points}

Topical imiquimod is off-label treatment for lentigo maligna when surgery cannot be performed

Imiquimod 5\% cream is applied once daily for 5 days per week up to 12 weeks with a $1-2 \mathrm{~cm}$ margin of clinically normallooking skin

The effect of imiquimod is mediated by inflammation leading to erythema, edema, erosions, oozing and discomfort, which can be discouraging and lead patients to stop treatment

Our case shows that, regardless of interval treatment, complete clinical clearance of the lesion can be achieved without recurrence during 2-year follow-up

Interval treatment with imiquimod can be used in patients with severe inflammatory response and allow achieving a cumulative dose of a total of 12 weeks' treatment with a minimum of 60 applications

\section{INTRODUCTION}

Lentigo maligna (LM) is a type of melanoma in situ on sun-damaged skin of elderly patients [1]. The treatment of choice is excisional biopsy followed by wide excision with margins $>5 \mathrm{~mm}$ $[1,2]$. Topical imiquimod is an off-label alternative treatment when surgery is not possible. The typical effect of imiquimod's action is a local inflammatory reaction, which may cause the patient to stop treatment [3]. We present a patient with lentigo melanoma on the face in close proximity to the eye, in whom interval treatment with topical imiquimod 5\% was carried out because of the severe inflammatory response.

\section{CASE REPORT}

An 82-year-old Caucasian woman presented with a large, irregularly pigmented lesion on her left cheek within the scar of a previously excised lesion (Fig. 1a). The previous lesion had been excised 6 years earlier with the margins remaining clear. The histopathological diagnosis was a junctional nevus. According to the patient's information, a few months after the surgery the lesion had started to reappear. Clearly this clinical course was against a nevus diagnosis, leading to suspicion of lentigo maligna or melanoma.

On clinical examination the lesion was localized on the upper part of the left cheek, had a triangular shape with irregular borders, and was multicolored with brown, black, and gray. The videodermatoscopy revealed an asymmetric pigmentation with gray dots around the follicles, rhomboid structures, and obliteration of the follicular openings (Fig. 2a, b).

A shave biopsy of the lesion was performed. The histopathological examination showed a melanocytic lesion with junctional and intraepidermal proliferation of single melanocytes with little tendency to grouping into small nests. Melanin content in the epidermis was uneven (Fig. 3a). The melanocytes presented moderate features of atypia such as pleomorphic and hyperchromatic nuclei. Melanocytes invaded the upper part of the hair follicle epithelia. There were scattered melanophages underneath the epidermis in the papillary dermis and solar elastosis indicating sun-damaged skin (Fig. 3b). The diagnosis of lentigo maligna was established.

Surgical treatment was abandoned because of the large size of the lesion, risk of disfigurement, and the patient's refusal of this treatment modality. Since we did not want to leave the patient untreated, treatment with imiquimod $5 \%$ once daily, five times per week, was proposed. After 5 weeks the treatment with imiquimod was stopped because of intense inflammation and crusted ulceration (Fig. 1b). After 3 months, on the follow-up visit videodermatoscopy was performed and showed 

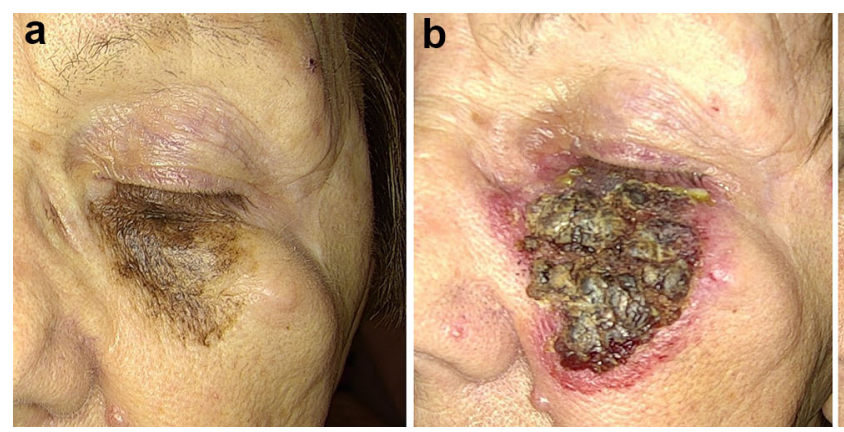

Fig. 1 Clinical presentation of the facial lesion. a Lentigo maligna before treatment. $\mathbf{b}$ Response to imiquimod with severe inflammatory reaction after 5 weeks of treatment.
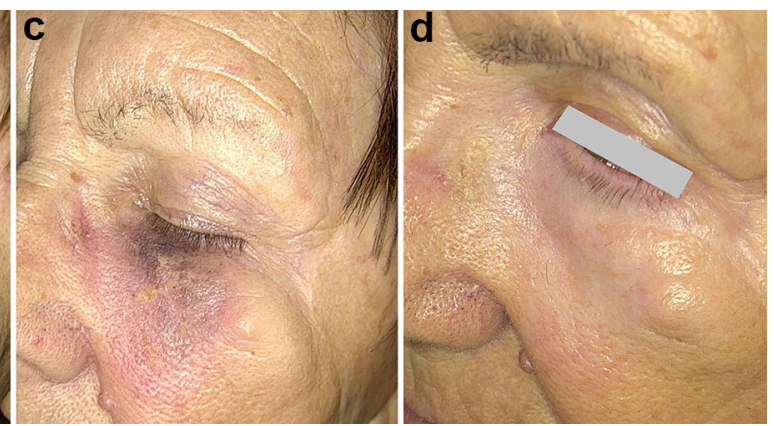

c Effect after the first course of imiquimod when oozing and crusting resolved. $\mathbf{d}$ Clinical effect after 2-year followup
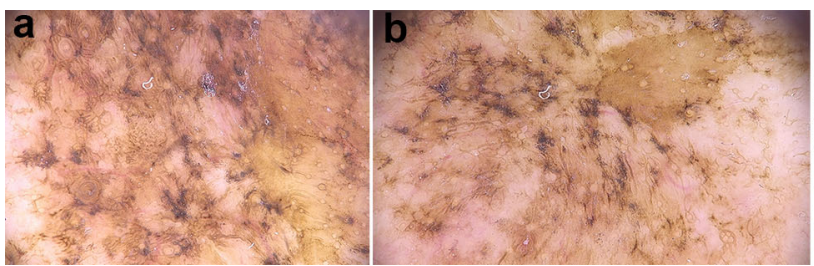

Fig. 2 Videodermatoscopy of the lesion. a, b Dermatoscopical images of lentigo maligna before the treatment showed an asymmetric pigmentation with gray dots around the follicles, rhomboid structures, and obliteration of the follicular openings (magnification $\times 20$ ). c Dermatoscopical presentation of the lesion after the first course of imiquimod showed changes in the pigmentation,
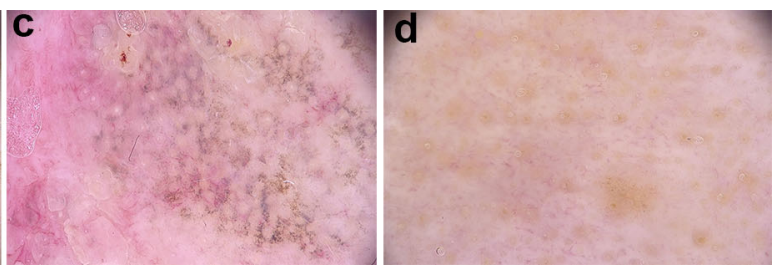

residual gray dots, and fading of the previously present dark brown and black colors (magnification $\times 20$ ). $\mathbf{d}$ Presentation of the affected skin after 2-year follow-up. Hair follicle openings are seen but there is no pigment in and around them. Also, the interfollicular skin has normal skin color (magnification $\times 20$ )

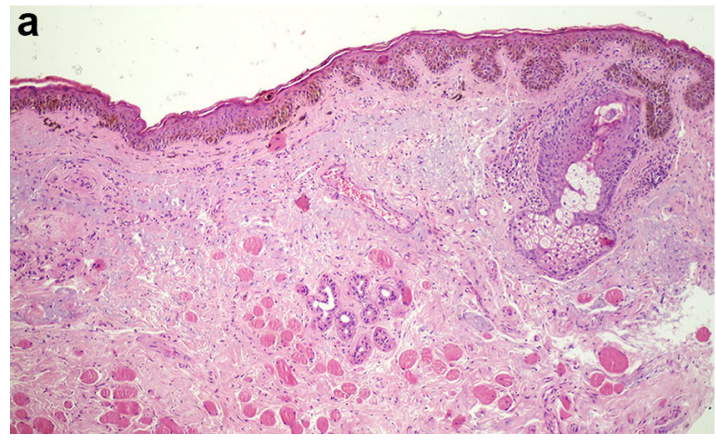

Fig. 3 Histopathology of the pigmented lesion. a, b Histopathological examination before the treatment revealed junctional and intraepidermal proliferation of single melanocytes with little tendency to group into nests and uneven melanin production. Melanocytes presented

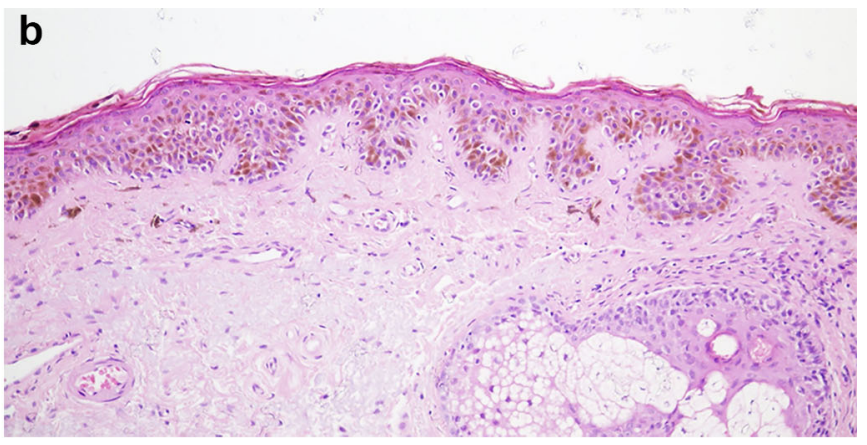

moderate atypia such as pleomorphic and hyperchromatic nuclei. They invaded the upper part of the hair follicle epithelium. In the dermis there was solar elastosis with scattered melanophages pointing to chronically sun-damaged skin $(\mathbf{a} \times 100 ; \mathbf{b} \times 200$ magnification $)$ 
changes in the pigmentation in the lentigo maligna lesion including the presence of residual gray dots and fading of the previously present dark brown and black colors. The inflammation response had almost resolved (Figs. 1c, 2c). The second course of the treatment with imiquimod five times per week was introduced again for 5 weeks, and after 2 months the third course with the same regimen was repeated. After the second and third courses, the inflammation was less intense. The duration of intervals between the courses was adapted to the patient's demands.

Total clearance of the lesion was achieved, which was confirmed by dermatoscopical examination. Hair follicle openings were seen but there was no pigment in and around them. Also, the interfollicular skin had normal skin color. During 2-year follow-up, no relapse of the lesion was observed (Figs. 1d, 2d).

The patient gave oral and written informed consent for the publication of this case including clinical photographs.

\section{DISCUSSION}

Lentigo maligna is a melanoma in situ on sundamaged skin. It usually presents on the head and neck of elderly patients with chronically sun-damaged skin [1]. LM is usually a slowgrowing melanoma; however, it may progress to invasive melanoma (lentigo maligna melanoma, LMM) [1]. In one study the risk of progression from LM to LMM was estimated as $3.5 \%$ (95\% CI 2.5-5.0) per year [4]. Lentigo maligna and lentigo maligna melanoma represent $4-15 \%$ of all melanomas [1]. According to international guidelines, surgery is the treatment of choice. Due to the risk of subclinical extension, LM may require wider excision with margins $>5 \mathrm{~mm}$, which can be challenging for facial lesions [2]. The need for wider excision of lentigo maligna is due to its high rate of local recurrence of all melanoma subtypes when treated by standard surgical excision. This rate of recurrence is $20 \%$.

The treatment of lentigo maligna with imiquimod can offer off-label and alternative cure methods in cases when surgery carries the risk of severe disfigurement or is contraindicated. There is no approved or recommended treatment regimen. There are several reports of imiquimod's therapeutic usefulness for lentigo maligna before instead of after surgery [5-7]. Usually the $5 \%$ cream is applied once daily $5-$ 7 days per week for 12 weeks with a $1-2-\mathrm{cm}$ margin of clinically normal-looking skin [1]. There are studies showing that a cumulative dose of at least 60 applications for $\geq 5$ days per week increases the efficacy of treatment $[8,9]$. The clinical response of imiquimod monotherapy ranges from 37 to $78 \%[3,9]$, with recurrence rates reported in $2.2-10.5 \%$ of cases $[6,9]$. The mean time to recurrence was over 4 years.

Imiquimod's mode of action is based on innate and acquired immune system stimulation leading to erythema, edema, oozing and erosion formation [10]. All these features cause discomfort to the patient, but they are indicators of the therapeutic response. In a systematic review by Tio et al., 43 of $509(8.4 \%)$ patients dropped out during the treatment because of inflammation or unrelated causes or were lost to follow-up [9]. In another study, 3 of 57 (5.3\%) patients stopped the treatment because of the aforementioned side effects [6].

Severe inflammatory response causing patient pain and reduction of quality of life is a limitation of imiquimod treatment. Our patient also experienced these, and we decided to stop imiquimod application until the patient recovered. Due to the intensive inflammatory reaction, the treatment was divided into three courses (15 weeks of total treatment, 75 applications). The inflammation after the second and third courses was less intense. We have not changed the recommendations for imiquimod application. We do not know whether the weaker inflammatory response was due to the partial destruction of the lesion or to application a smaller amount of the $5 \%$ cream.

Dividing the treatment into shorter courses reduces the risk of side effects, giving the possibility to achieve a cumulative dose of a total of 12 weeks' treatment with a minimum of 60 applications. We called this interval treatment. In our case, treatment interruptions were tailored to the personal needs of the patient and were longer than the time needed for the 
wound healing. Further studies are needed to assess the optimal break time between courses in patients with severe inflammatory responses.

Total clearance of the pigmented lesion was achieved, despite the initial severe inflammatory reaction and ulceration. At present, the patient is having regular follow-up controls every 6 months.

\section{CONCLUSION}

Interval treatment of lentigo maligna with 5\% topical imiquimod can help to overcome the severe inflammatory reaction patients may experience. However, the presence of the severe inflammatory reaction is a clinical indication of the efficacy of this therapeutic approach. Malignant melanocytes are destroyed by activated lymphocytes and secreted cytokines, and this is a mechanism of imiquimod action. Our case shows that even with interval treatment, complete clinical clearance of the lesion was achieved with no recurrence during 2 years of follow-up.

\section{ACKNOWLEDGEMENTS}

We thank the participants in the study.

Funding. The Journal's Rapid Service Fee was covered by Medical University of Warsaw (1M4/1/PUR/N/21).

Authorship. All named authors meet the International Committee of Medical Journal Editors (ICMJE) criteria for authorship for this article, take responsibility for the integrity of the work as a whole, and have given their approval for this version to be published.

Author Contributions. MM-G, JC and AG consulted the patient; MM-G and JC performed histopathological evaluation, lead conceptual work and drafted the manuscript; AC collected the literature; AG, AC and LR corrected the manuscript, and all authors read and accepted the final version of the article.
Disclosures. Magdalena Misiak-Galazka, Joanna Czuwara, Adam Galazka, Anna Czaplicka and Lidia Rudnicka all have nothing to disclose.

Compliance with Ethics Guidelines. The patient gave oral and written informed consent for the publication including clinical photographs.

Open Access. This article is licensed under a Creative Commons Attribution-NonCommercial 4.0 International License, which permits any non-commercial use, sharing, adaptation, distribution and reproduction in any medium or format, as long as you give appropriate credit to the original author(s) and the source, provide a link to the Creative Commons licence, and indicate if changes were made. The images or other third party material in this article are included in the article's Creative Commons licence, unless indicated otherwise in a credit line to the material. If material is not included in the article's Creative Commons licence and your intended use is not permitted by statutory regulation or exceeds the permitted use, you will need to obtain permission directly from the copyright holder. To view a copy of this licence, visit http://creativecommons.org/licenses/bync/4.0/.

\section{REFERENCES}

1. Iznardo H, Garcia-Melendo C, Yélamos O. Lentigo maligna: clinical presentation and appropriate management. Clin Cosmet Investig Dermatol. 2020;13:837-55

2. Swetter SM, Tsao H, Bichakjian CK, et al. Guidelines of care for the management of primary cutaneous melanoma. J Am Acad Dermatol. 2019;80:208-50.

3. Hyde MA, Hadley ML, Tristani-Firouzi P, Goldgar D, Bowen GM. A randomized trial of the off-label use of imiquimod, 5\%, cream with vs without tazarotene, $0.1 \%$, gel for the treatment of lentigo maligna, followed by conservative staged excisions. Arch Dermatol. 2012;148:592-6.

4. Menzies SW, Liyanarachchi S, Coates E, et al. Estimated risk of progression of lentigo maligna to 
lentigo maligna melanoma. Melanoma Res. 2020;30:193-7.

5. Lallas A, Moscarella E, Kittler H, et al. Real-world experience of off-label use of imiquimod 5\% as an adjuvant therapy after surgery or as a monotherapy for lentigo maligna. Br J Dermatol. 2021;185:675-7.

6. Tio D, van Montfrans C, Ruijter CGH, Hoekzema R, Bekkenk MW. Effectiveness of 5\% topical imiquimod for lentigo maligna treatment. Acta Derm Venereol. 2019;99:884-8.

7. Kirtschig G, van Meurs T, van Doorn R. Twelveweek treatment of lentigo maligna with imiquimod results in a high and sustained clearance rate. Acta Derm Venereol. 2015;95:83-5.
8. Mora AN, Karia PS, Nguyen BM. A quantitative systematic review of the efficacy of imiquimod monotherapy for lentigo maligna and an analysis of factors that affect tumor clearance. J Am Acad Dermatol. 2015;73:205-12.

9. Tio D, van der Woude J, Prinsen CAC, Jansma EP, Hoekzema R, van Montfrans C. A systematic review on the role of imiquimod in lentigo maligna and lentigo maligna melanoma: need for standardization of treatment schedule and outcome measures. J Eur Acad Dermatol Venereol. 2017;31:616-24.

10. Schön MP, Schön M. Imiquimod: mode of action. Br J Dermatol. 2007;157(Suppl 2):8-13. 